\title{
Temperature Effects on the Atomic Structure and Kinetics in Single Crystal Electrochemistry
}

\author{
Yvonne Gründer $^{1 *}$, Nenad M. Markovic ${ }^{2}$, Paul Thompson ${ }^{1}$ and Christopher A. Lucas ${ }^{1 *}$ \\ ${ }^{1}$ Oliver Lodge Laboratory, Department of Physics, University of Liverpool, Liverpool, \\ L69 7ZE, United Kingdom \\ ${ }^{2}$ Materials Science Division, Argonne National Laboratory, Argonne, IL 60439, USA
}

*Corresponding authors: grunder@liv.ac.uk, clucas@liv.ac.uk

\begin{abstract}
The influence of temperature on the atomic structure at the electrochemical interface has been studied using in-situ surface X-ray scattering (SXS) during the formation of metal monolayers on a $\mathrm{Au}(111)$ electrode. For the surface reconstruction of $\mathrm{Au}(111)$, higher temperatures increase the mobility of surface atoms in the unreconstructed phase which then determines the surface ordering during the formation of the reconstruction. For the underpotential deposition (UPD) systems, the surface diffusion of the depositing metal adatoms is significantly reduced at low temperatures which results in the frustration of ordered structures in the case of $\mathrm{Cu} U \mathrm{PD}$, occurring on a $\mathrm{Br}$-modified surface, and in the formation of a disordered $\mathrm{Ag}$ monolayer during $\mathrm{Ag}$ UPD. The results indicate that temperature changes affect the mass transport and diffusion of metal adatoms on the electrode surface. This demonstrates the importance of including temperature as a variable in studying surface structure and reactions at the electrochemical interface.
\end{abstract}


Keywords: Electrochemistry; Electrodeposition; Gold; Surface Structure; X-ray scattering 


\section{Introduction}

Since the early days of modern surface science, the main goal in the electrochemical community has been to find correlations between the microscopic structures formed by surface atoms and adsorbates and the macroscopic kinetic rates of a particular electrochemical reaction. Single crystal surface electrochemistry has advanced this field of research through the ability to control the surface atomic structure and, hence, the nature of the surface sites for adsorption of species from the electrolyte side of the interface. The development of in-situ surface-sensitive probes, most notably synchrotron-based surface x-ray scattering (SXS), scanning tunneling microscopy (STM), Raman spectroscopy and Fourier Transform Infrared (FTIR) spectroscopy [1-3] have enabled the transformation of electrochemistry from a largely phenomenological subject into a discipline that addresses atomic/molecular-level issues in a detail that is comparable to that achieved in ultra-high vacuum (UHV) based surface science [4]. Of particular interest have been potential-driven phenomena at ambient temperatures, ranging from ordering, phase transitions and mobility of both metal surface atoms $[5,6]$ and adsorbed layers [7-10] to the surface compositions and segregation profiles of bimetallic systems [11]. SXS experiments have been crucial in the advances that have been made, as the technique gives information about the atomic structure at the surface of the solid electrode and the composition of the sub-surface region, the latter of which can be crucial in determining the reactive properties of the surface. In addition SXS can also provide information about ordering in the 'double-layer' close to the electrode surface [12]. The application of SXS to study the structure at the electrochemical interface has been described in a number of review articles $[4,13,14]$. Despite the advances in surface electrochemistry, in practical applications many relevant 
electrochemical processes occur at non-ambient temperatures and yet little is known about the effect of temperature on the atomic structure at the electrochemical interface. Whereas in-situ imaging techniques are difficult at high temperature, the SXS technique only requires the modification of the SXS electrochemical cell to control the temperature of the electrolyte during the SXS measurements. Measurements that were obtained in an electrochemical SXS cell that operates over the temperature range 275$325 \mathrm{~K}$ were previously reported and showed that temperature can have a strong influence on both restructuring at the interface, i.e. the potential-dependent surface reconstruction of $\mathrm{Au}(100)$, and on potential-driven reactions, such as the adsorption and oxidation of $\mathrm{CO}$ on a $\mathrm{Pt}(111)$ electrode [15]. The results suggested that the main effect of temperature is on the kinetics of oxide formation, the activation of water and the adsorption of oxygenated species. The importance of temperature in controlling interfacial properties goes well beyond the aqueous environment. Electrochemistry in non-aqueous electrolytes, such as organic electrolytes and ionic liquids, is becoming of increasing importance and these electrolytes offer a significantly increased temperature range. Thus the importance of establishing a methodology for temperature dependent studies is clear.

In this paper we present further studies of the influence of temperature on the structure and kinetics of metal monolayer formation at the $\mathrm{Au}(111)$ electrode surface. First we describe the effects on the potential-dependent surface reconstruction of $\mathrm{Au}(111)$, a topic that is well studied in both UHV [16] and electrochemical surface science $[6,17]$. In UHV, the surface reconstructions of $\mathrm{Au}$ are stable up to high temperatures, whereas in the electrochemical environment the state of the surface can be controlled by the electrode potential via the strong metal-adsorbate interaction and/or 
the electrode surface charge [18]. The potential dependent dynamics of the hexagonal reconstruction (hereafter denoted as "hex") of the $\mathrm{Au}(111)$ and $\mathrm{Au}(100)$ electrode surfaces, the transitions to the $(1 \mathrm{x} 1)$ phases and the attendant mass transfer have been of fundamental significance for some decades. The structural behavior is further enriched with the adsorption of $\mathrm{CO}$ which, surprisingly, acts as a "guardian" of the hex phase. It has also been proposed that adsorbed carbon monoxide can act as a promoter on these electrode surfaces for the electrocatalytic oxidation of certain alcohols [19]. In the first part of the paper the temperature dependence of the formation of the 'hex' reconstruction of $\mathrm{Au}(111)$ in alkaline electrolyte $(0.1 \mathrm{M} \mathrm{KOH})$ is explored. The remainder of the paper describes the effects of temperature on the formation of bimetallic surfaces through the process of underpotential deposition (UPD), namely $\mathrm{Cu}$ UPD onto $\mathrm{Au}(111)$ in the presence of bromide anions and Ag UPD onto $\mathrm{Au}(111)$. The influence of bromide anions in controlling and stabilizing the Cu UPD layer on different single crystal electrodes has been explored in some detail, including a paper in the last Surface Science special issue dedicated to electrochemistry [20]. The formation of an ordered $\mathrm{Cu}-\mathrm{Br}$ bilayer structure with a symmetry that differs from the underlying $\mathrm{Au}(111)$ surface allows the SXS signal from the bilayer structure to be measured independently so that detailed structural information about the ordering process can be obtained. The deposition of a Ag UPD monolayer onto $\mathrm{Au}(111)$, in which a commensurate Ag monolayer is formed, is studied by measurement of crystal truncation rod (CTR) data.. It is shown that temperature influences the mass transport and surface adatom diffusion during the UPD process, in turn, strongly effecting the thin film morphology. Finally, we summarize the results and offer some perspective for future studies of temperature effects in electrochemical environments. 


\section{Experimental Section}

The $\mathrm{Au}(111)$ single crystal $\left(\right.$ miscut $<0.1^{\circ}$ ) was prepared by sputtering and annealing in a UHV system for several days until a sharp LEED pattern was observed. The sample was then removed from UHV and, prior to the x-ray measurements, was annealed using a butane flame and cooled in air. The crystal was then transferred to the electrochemical $\mathrm{x}$-ray cell with a drop of ultra-pure water protecting the surface and was immersed at open circuit potential in electrolyte. Following these procedures the crystal surface remains free from contaminants, as confirmed by the electrochemical response, which is sensitive to impurity concentrations. The experimental procedure followed that of similar studies reported previously [4]. X-ray measurements were performed on BM28 (XMaS), the UK-CRG beamline at the ESRF, Grenoble, the surface x-ray diffraction beamline, ID3, at the ESRF, Grenoble, beamline BM12-BESSRC at the APS, Argonne National Laboratory and beamline 7-2 at the Stanford Synchrotron Radiation Laboratory (SSRL). Full details of the experimental setups used at each of the beamlines, e.g. details of the diffractometer, slit settings and relevant resolution corrections, can be found in references [15, 21-24]. The $\mathrm{Au}(111)$ crystal was indexed to a conventional hexagonal unit cell for a $f c c(111)$ surface where the surface normal is along the $(0,0, \mathrm{~L})_{\text {hex }}$ direction and the $(\mathrm{H}, 0,0)_{\text {hex }}$ and $(0, \mathrm{~K}, 0)_{\text {hex }}$ directions lie in the surface plane and subtend $60^{\circ}$. In this case the units for $\mathrm{H}, \mathrm{K}$ and $\mathrm{L}$ are $a^{*}=b^{*}=$ $4 \pi / \sqrt{ } 3 a_{\mathrm{NN}}$ and $c^{*}=2 \pi / \sqrt{ } 6 a_{\mathrm{NN}}$ respectively, where $a_{\mathrm{NN}}$ is the nearest-neighbor distance in the crystal $\left(a_{\mathrm{NN}}=2.885 \AA\right.$ for $\left.\mathrm{Au}\right)$. The outer chamber of the x-ray cell was continuously purged with nitrogen to protect the surfaces from oxygen. The reference electrode used in the x-ray cell was a saturated calomel electrode (SCE). To facilitate comparison to 
previous results and publications only the underpotential deposition of copper (section 3.2) is quoted against SCE. For $\mathrm{Au}(111)$ in $0.1 \mathrm{M} \mathrm{KOH}$ (section 3.1) the potentials are quoted versus the reversible hydrogen electrode (RHE) and the data for $\mathrm{Ag}$ underpotential deposition are quoted versus $\mathrm{a}_{\mathrm{Ag}^{+}}$reference. Any shifts due to temperature changes are relatively small (of the order of $0.01 \mathrm{~V}$ ) over the temperature range of study. A schematic of the SXS electrochemical cell, which has been modified from previous designs to allow temperature control, is shown in Figure 1. The temperature of the electrochemical cell (made from Macor) was controlled using a water-cooled Peltier device at the base of the cell and monitored using a thermocouple embedded in the wall of the cell close to the sample surface. Although precise temperature readings are difficult in this arrangement we estimate that the quoted values are accurate to $\pm 3^{\circ}$. All of the measurements were made following the same methodology; following a temperature change, the potential was cycled before any of the results presented in this paper were measured. This eliminates any influence of surface preparation on the results obtained so that they are representative of equilibrium conditions. All results presented are reversible with temperature.

\section{Results and Discussion}

\subsection{The Surface Reconstruction of $\mathrm{Au}(111)$}

The Au (111) surface reconstruction has been shown to exhibit the well-known ( $\mathrm{x} \times \sqrt{3}$ ) phase using a variety of in situ methods including STM [25, 26], and SXS[27]. Numerous UHV studies have also been performed using LEED [28], surface x-ray diffraction [29], and STM [30] techniques. More recently, advanced computational simulations have been performed in an attempt to try and obtain a more fundamental 
understanding of the surface reconstruction [31]. Whilst the $(p \times \sqrt{3})$ reconstructed phase is widely accepted in both UHV and electrolyte, the exact nature of the surface behavior is yet to be fully resolved. For example, the ordered array of kinks from UHV measurements (the so called "chevron phase) [29], is not detected by x-rays when the surface is in contact with electrolyte [27]. In this latter case the initial morphology of the $\mathrm{Au}(111)$ surface plays an important part in the reconstruction process.

The effect of temperature on the cyclic voltammetry of $\mathrm{Au}(111)$ in $0.1 \mathrm{M} \mathrm{KOH}$ has been studied previously where it was shown that by increasing temperature : (i) the peak at $\sim 1.25 \mathrm{~V}$, corresponding to surface oxide formation, is shifted negatively [32]; and (ii) the adsorption of hydroxyl ions $\left(\mathrm{OH}_{\mathrm{ad}}\right)$ becomes more reversible. In line with our previous studies [33, 34] and STM data [35], the formation of a "true" oxide by irreversible adsorption of oxygenated species has a profound effect on variations in the surface morphology of $\mathrm{Au}$ [36]. For the specific case of Au(111), STM images have shown that, after oxide formation at $\sim 1.3 \mathrm{~V}$, on the reverse potential sweep monoatomic deep "pits" are formed across the surface due to both some dissolution of Au atoms and repositioning of surface atoms after the oxide reduction [37]. It is reasonable to anticipate that at higher temperatures any morphological changes due to oxideformation/oxide-reduction cycles will be amplified because the nucleation of the reconstruction will be occurring on a surface with varying amounts of defects. Concomitantly, the surface reconstruction formed at different temperatures should reflect this.

To probe the effect of temperature, the $\mathrm{Au}(111)$ electrode was studied at $281 \mathrm{~K}$ (low temperature, LT), $299 \mathrm{~K}$ (ambient temperature, AT) and $313 \mathrm{~K}$ (high temperature, HT) in $0.1 \mathrm{M} \mathrm{KOH}$. These results are summarized in Figure 2. Figure 2(a) shows scans 
along the $\langle 1,1,0\rangle$ direction through the $(0,1,0.52)$ reciprocal lattice position measured at $-0.75 \mathrm{~V}$, a potential where the surface is fully reconstructed, at two different temperatures. Two peaks are observed (i) the peak at $\mathrm{H}=0, \mathrm{~K}=1$ corresponding to the scattered x-ray signal at $(0,1,0.52)$, an anti-Bragg position on the $(0,1, \mathrm{~L})$ crystal truncation rod (CTR) and (ii) a peak at $\mathrm{H} \sim 0.02, \mathrm{~K} \sim 1.02$ which arises due to the $\mathrm{Au}$ $(p x \sqrt{3})$ surface reconstruction. From the scans in Figure 2(a), it appears that at low temperature there is a subtle difference in the nature of the reconstructed surface. Fits to the scans (the solid lines which correspond to a double Lorentzian lineshape with a linear sloping background), indicate that the integrated intensity, which represents the amount of the surface that is reconstructed, is lowest at low temperature $(281 \mathrm{~K})$. There is no statistically significant difference between the measurements taken at ambient temperature ( $299 \mathrm{~K}$, data not shown) and high temperature $(313 \mathrm{~K})$. Also, the shift in peak position from LT to AT (from the fits to the data $\mathrm{H}=0.0208$ (LT), and $\mathrm{H}=0.0216$ (HT)), showed that at HT (and AT) the surface was more compressed than at LT, corresponding to $p$ values of $23 \pm 0.1$ and $24 \pm 0.1$ respectively. It should be noted that during the 'grooming' of the $\mathrm{Au}(111)$ surface in $0.1 \mathrm{M} \mathrm{KOH}$, by repeated potential cycles over the potential range shown, there is a gradual reduction in the value of $p$ as the surface orders until it saturates at $\mathrm{p} \sim 23$ which indicates that the surface compression is directly correlated with the number of surface defects. The difference in the fitted peak widths indicates that, at both AT and HT, the reconstruction domain size is slightly larger compared to LT. In addition to the data shown in Figure 2(a), potentiodynamic measurements, so called $\mathrm{x}$-ray voltammetry (XRV) in which the $\mathrm{x}$-ray intensity at a structure-sensitive CTR position is monitored as a function of the potential, were obtained at $(0,1,0.5)$ at the three different temperatures quoted, to investigate the effect 
of temperature on the $\mathrm{Au}(111)-(1 \mathrm{x} 1) \rightarrow \mathrm{Au}(111)-(\mathrm{px} \sqrt{3})$ transition. The positive XRV sweeps, shown in Figure 2(b), correspond to the lifting of the reconstruction where the intensity decreases slightly during the lifting of the reconstruction before increasing when the surface orders in the (1x1) phase. On the negative sweep, the surface morphology is changing due to the reduction of reversible oxide formed at the positive limit, followed by the phase change to the $(p x \sqrt{3})$ phase. The effect of reducing the temperature appears to slow down the kinetics of the reconstruction process, i.e. the data at low temperature is 'smeared out' over a broader potential window and to decrease the ordering of the reconstructed layer. For reconstruction of $\mathrm{Au}(111)$ the mass transport is fairly small ( 4\%), which reflects the small difference in surface metal atomic density between the reconstructed and unreconstructed surfaces. In contrast, for $\mathrm{Au}(001)$ the transition between the "hex" and $(1 \mathrm{x} 1)$ phase, observed under the same conditions as for $\mathrm{Au}(111)$, involves a substantial $(\sim 20 \%)$ change in surface metal atomic density. It is thus not surprising that the effect of temperature changes on the potential-induced reconstruction of $\mathrm{Au}(001)$ is rather more dramatic [15] .

In addition to the potential-dependent surface reconstruction of gold, in the last few decades there have been substantial advances in understanding the catalytic properties of gold both in the gas phase and electrochemical environments [38-40]. For example, it has recently been demonstrated by Rodriguez, Koper and co-workers that adsorbed CO may have a promoting effect on the oxidation of alcohols [19, 41-43]. The authors proposed that adsorbed $\mathrm{CO}$ can lead to enhanced adsorption of $\mathrm{OH}_{\mathrm{ad}}$ at negative potentials which, in turn, promotes the oxidation of alcohol reactive intermediates [41]. The fact that the effect is only observed on the hexagonal close-packed surfaces of Au suggests that there is a fundamental link between the adsorption of $\mathrm{CO}$ and the surface 
structure. Recently we have studied the atomic structure of the Au(111) surface by SXS, both in alkaline solution in the presence of $\mathrm{CO}$ and during the oxidation of ethanol and methanol [44]. The compression of the surface and the presence of defects are found to play a key role in the electrocatalytic activity, as the products of alcohol oxidation and their adsorption properties determine both the potential range over which the reconstruction is stable and the diffusion of the excess surface Au atoms during the lifting of the reconstruction. In combination with the measurements presented here it is clear, therefore, that not only may the activity of the Au surface be influenced by the adsorption of species from electrolyte but that the defect concentration and surface compression could potentially also be controlled by the temperature. Thus in the future it may be possible to decouple the effects of molecular adsorption and the role of defects on the enhanced electrocatalytic activity. Experiments probing the effects of temperature on the oxidation of alcohols would give fundamental insight into this process.

\subsection{The Underpotential Deposition of $\mathrm{Cu}$ in the Presence of $\mathrm{Br}$ anions}

The phenomenon of underpotential deposition (UPD) on metal surfaces has been widely studied and is of importance for both a fundamental understanding of metal deposition as well as for electrocatalysis on bimetallic surfaces. In particular, the role of halide anions on the surface structure of UPD adatoms and the kinetics of metal deposition has received considerable attention. In addition, the role of halide anions in stabilizing UPD monolayers has been illustrated both by experimentalists [20, 45-51] and theoreticians alike [52-54]. Despite this interest, there has been little progress in understanding partial charge transfer at the electrochemical interface although it has 
been postulated that the adsorption of anions and, especially, partial charge transfer is strongly affected by the presence and strength of water adsorption at a given potential. Furthermore, the effect of changing the temperature during UPD has received little attention despite the fact that electroplating processes are often performed at nonambient temperature.

The UPD of $\mathrm{Cu}$ onto both $\mathrm{Au}(111)$ and $\mathrm{Pt}(111)$ electrodes has been studied in detail by a combination of cyclic voltammetry (including rotating ring disc electrode measurements) and SXS [48-50, 55, 56]. In both cases Cu UPD occurs when the surface is covered by a close-packed bromide monolayer and the structures that are formed during the UPD process are governed by the ordering in the halide adlayer. Although the bromide adlayer that is formed at anodic potentials on $\mathrm{Au}(111)$ forms an incommensurate structure that is rotated with respect to the underlying $\mathrm{Au}$ lattice, $\mathrm{Cu}$ UPD transforms the adlayer into a hexagonal structure that is aligned with the Au lattice. As the potential is scanned cathodically the $\mathrm{Cu}-\mathrm{Br}$ adlayer structure is incommensurate over a narrow potential range and undergoes compression before locking into a structure with $(4 \mathrm{x} 4)$ symmetry, i.e. the near-neighbour spacing in the $\mathrm{Cu}$ Br layer is $3.847 \AA$ and the principal diffraction peak is observed at $(0.75,0, \mathrm{~L})$ and $60^{\circ}$ rotationally equivalent positions. A schematic of the surface plane of the $\mathrm{Au}(111)$ reciprocal space is shown in Figure 3(a), which indicates where the scattering from the $\mathrm{Cu}-\mathrm{Br}$ adlayer is observed.

The structure of the $\mathrm{Cu}-\mathrm{Br}$ adlayer on $\mathrm{Au}(111)$ allows a detailed SXS measurement of its potential dependence to be made and the influence of the temperature effects to be examined closely. Measurements were made at low (279 K) and high $(311 \mathrm{~K})$ temperature in $0.1 \mathrm{M} \mathrm{H}_{2} \mathrm{SO}_{4}+1 \mathrm{mM} \mathrm{Cu}^{2+}+1 \mathrm{mM} \mathrm{KBr}$ and the results 
are shown in Figures 3 and 4. Figure 3(b) shows scans along the $\langle 1,1,0\rangle$ direction through the $\mathrm{Cu}-\mathrm{Br}$ adlayer peak at $70 \mathrm{mV}(\sim 200 \mathrm{mV}$ positive of the negative potential limit below which bulk $\mathrm{Cu}$ deposition occurs) and at $370 \mathrm{mV}$ (close to the positive potential limit above which no ordered structure is observed). Figure 4 shows the XRV measured at the position $(0.745,0.745,0.2)$ and a summary of the results obtained by fits to the $(\mathrm{H}, \mathrm{K})$ scans (for example the data shown in Figure 3(b)) as the potential was stepped across the entire potential range beginning at $450 \mathrm{mV}$ and ending at $-100 \mathrm{mV}$. Fits of a Lorentzian lineshape to the data (as shown in Figure 3(b)-solid lines) permit the peak position, domain size (proportional to the inverse of the FWHM of the peak) and peak amplitude to be obtained and these are shown in Figures 4(b), 4(c) and 4(d) respectively. The potentiodynamic data in Figure 4(a) immediately indicates that at high temperature the ordering in the $\mathrm{Cu}-\mathrm{Br}$ adlayer is significantly improved. This is supported by the results in Figure 4(c) and 4(d) which show that as the potential is stepped negatively there is considerable frustration in the ordering of the $\mathrm{Cu}-\mathrm{Br}$ adlayer at low temperature, particularly over the range $400 \mathrm{mV}$ down to $200 \mathrm{mV}$ at the onset of structure formation. Furthermore, although at high temperature the structure locks into the commensurate phase at $\sim 300 \mathrm{mV}$, at which point the domain size reaches a maximum value, at low temperature the commensurate $\mathrm{Cu}-\mathrm{Br}$ adlayer is not formed over the entire potential range and the amplitude of the peak is considerably smaller, indicative of a reduced surface coverage of the ordered phase.

Recently it has been shown experimentally that the presence of halide adlayers has a strong effect on the morphology of the metal electrodeposit formed during $\mathrm{Cu}$ homoepitaxy on a $\mathrm{Cu}(001)$ surface [57]. This was attributed to the influence of the halide adlayer on the surface energy landscape, particularly the effective change in 
dipole moment during adatom diffusion. In our initial study of temperature effects in electrochemistry it was demonstrated that temperature changes have little effect on the structure and kinetics of halide adlayers both on $\mathrm{Au}$ and Pt electrodes [15]. The temperature effects demonstrated in Figures 3 and 4 can thus be attributed mainly to the influence on the surface diffusion of the $\mathrm{Cu}$ adatoms during UPD. Although the structure of the $\mathrm{Cu}-\mathrm{Br}$ adlayer that is formed during UPD onto $\mathrm{Au}(111)$ is determined largely by the compact $\mathrm{Br}$ adlayer that is present over a range of $\mathrm{Cu}$ coverage, the precise interface structure is strongly influenced by temperature as it controls the diffusion of the $\mathrm{Cu}$ atoms beneath the compact halide adlayer. Thus, at low temperature, the diffusion of $\mathrm{Cu}$ adatoms is limited and the formation of the commensurate $\mathrm{Cu}-\mathrm{Br}$ adlayer is frustrated, presumably by the formation of disordered domains and domain walls. In contrast, at high temperature the $\mathrm{Cu}$ - $\mathrm{Br}$ adlayer shows a high degree of order both in the incommensurate and commensurate phases over the entire potential range of $\mathrm{Cu}$ deposition. This is consistent with previous results for $\mathrm{Cu}$ UPD on $\operatorname{Pt}(111)$ [58], where a difference in the transferred charge and in the cyclic voltammetry was observed for low and high temperature pointing to a change in the phase formation possibly due to the frustration at low temperatures. Given that there is evidence that the halide adlayer remains on the $\mathrm{Cu}$ surface, even during bulk $\mathrm{Cu}$ deposition, it is clear that temperature changes can be important in determining the morphology of a thin metal film formed by electrodeposition. This could be further explored in an effort to gain sub-monolayer control during metal electrodeposition.

\subsection{The Underpotential Deposition of Ag}


There have been numerous studies of the UPD of $\mathrm{Ag}$ onto $\mathrm{Au}(111)[47,59,60]$. These have shown that there are different potential regions for monolayer, bilayer and bulk deposition, hence electrochemical deposition can allow fine control of the Ag structure formed. The cyclic voltammetry (CV) for a $\mathrm{Au}(111)$ electrode in $0.05 \mathrm{M}$ $\mathrm{H}_{2} \mathrm{SO}_{4}+1 \mathrm{mM} \mathrm{Ag}_{2} \mathrm{SO}_{4}$ shows three cathodic peaks at $0.59 \mathrm{~V}, 0.21 \mathrm{~V}$ and $0.1 \mathrm{~V}$ which are due to the deposition of $\mathrm{Ag}$. The peak at $0.1 \mathrm{~V}$ corresponds to deposition of a $\mathrm{Ag}$ bilayer which occurs at a potential just positive of the Nernst potential for bulk Ag deposition. Although the charge of the first two cathodic peaks corresponds to 0.72 of a Ag monolayer, Kondo et al. found using x-ray diffraction that the monolayer is actually complete at $0.12 \mathrm{~V}$ (vs $\mathrm{Ag} / \mathrm{AgCl}$ ). Interestingly, removal of the $1 \mathrm{ML} \mathrm{Ag} / \mathrm{Au}(111)$ electrode from the supporting electrolyte caused the Ag monolayer to adopt a partial bilayer structure which implies that the sulfate anions in solution stabilize the monolayer thin film morphology [61].

In order to probe the effects of temperature on the formation of the Ag UPD monolayer we performed SXS measurements at high $(311 \mathrm{~K})$ and low $(279 \mathrm{~K})$ temperature in $0.05 \mathrm{M} \mathrm{H}_{2} \mathrm{SO}_{4}+1 \mathrm{mM} \mathrm{AgSO}_{4}$ on a $\mathrm{Au}(111)$ electrode. The $\mathrm{XRV}$ measurements made at $(0,0,1.52)$, the anti-Bragg position on the specular CTR, are shown in Figure 5(a). Due to the fact that the atomic form factor for Ag is significantly smaller than that of $\mathrm{Au}$, deposition of $\mathrm{Ag}$ onto the $\mathrm{Au}(111)$ surface causes a decrease in the scattered intensity at the anti-Bragg positions of the CTRs, i.e. a full commensurate Ag monolayer effectively appears as a partial Au monolayer and hence the SXS signal at these CTR positions is reduced. As can be seen from the XRV data in Figure 5(a), Ag UPD causes a decrease in the scattered signal at the onset of Ag deposition ( 0.59 V) for both high and low temperature. As the potential is scanned negatively, however, at 
low temperature the signal begins to increase whereas at high temperature there is a further decrease in the scattered $\mathrm{x}$-ray signal. This is a clear sign that the temperature is having a significant effect on the morphology of the $\mathrm{Ag}$ film.

Figure 5(b) shows measurements of the specular CTR made after deposition of the Ag monolayer at low and high temperature recorded at a potential of $400 \mathrm{mV}$. The data are fully consistent with the XRV data indicating that at high temperature there is a significant decrease in the scattered signal at the anti-Bragg positions. This is emphasized in a plot of the ratio of the two CTR data sets shown in Figure 5(c). A simple atomic model was used to obtain the calculated fits to the CTR data shown by the solid lines which comprised of a single Ag monolayer with a variable coverage, $\mathrm{Au}-$ Ag surface normal spacing, $\mathrm{d}_{\mathrm{Ag} \text {-Au }}$, and a static Debye Waller factor, $\sigma$, representing a distribution of $\mathrm{d}_{\mathrm{Ag}-\mathrm{Au}}$. The results indicate that the coverage of the $\mathrm{Ag}$ monolayer, $\theta=1.0 \pm 0.1$, is identical at both temperatures and that the underlying $\mathrm{Au}$ surface is unreconstructed, i.e. consistent with the deposition of a complete Ag monolayer. The differences in the CTR data are described by changes in $d_{A g-A u}$ and $\sigma$; the high temperature results giving $\mathrm{d}_{\mathrm{Ag}-\mathrm{Au}}=2.39 \pm 0.06 \AA, \sigma=0.06 \pm 0.02 \AA$ and the low temperature results, $\mathrm{d}_{\mathrm{Ag}-\mathrm{Au}}=2.06 \pm 0.22 \AA, \sigma=1.3 \pm 0.2 \AA$. Although the fits to the CTR data with this simple model are not perfect they describe the essential differences in the morphology of the deposited Ag film, indicating substantial disorder in the structure formed at low temperature. In agreement with the studies of $\mathrm{Cu}$ UPD described in section 3.2 , it is apparent therefore that the mobility of the metal adatoms during Ag UPD is severely restricted at low temperatures and hence a disordered $\mathrm{Ag}$ film is formed. It is interesting to note that, similarly to $\mathrm{Cu}$ UPD in sulfate electrolyte where the sulfate molecules stabilize an open $\mathrm{Cu}$ honeycomb structure [62], the presence of sulfate stabilizes the $\mathrm{Ag}$ 
UPD layer. For example, transfer of the $\mathrm{Ag}(1 \mathrm{ML}) / \mathrm{Au}(111)$ electrode to $0.1 \mathrm{M} \mathrm{KOH}$ destabilizes the monolayer which reorders into a partial bi-layer structure [63]. It appears, therefore, that for Ag UPD, increasing the temperature only enhances the ability of sulfate to stabilize the Ag UPD layer.

\section{Conclusions}

In this paper, we have demonstrated that temperature changes can have dramatic effects on the atomic structure of electrochemical interfaces during the formation of metal monolayers. This was illustrated by SXS measurements of the Au(111) electrode during surface reconstruction, the UPD of $\mathrm{Cu}$ in the presence of bromide anions and the UPD of Ag. The principal effect of temperature is to control the mass transport and diffusion of metal adatoms on the electrode surface. Hence, for the surface reconstruction of $\mathrm{Au}(111)$, higher temperatures increase the mobility of surface atoms in the unreconstructed phase which then determines the surface ordering during the formation of the reconstruction. For the UPD systems, the surface diffusion of the depositing metal adatoms is significantly reduced at low temperatures which results in the frustration of ordered structures in the case of $\mathrm{Cu}$ UPD, occurring on a Br-modified surface, and in the formation of a disordered Ag monolayer during Ag UPD. In contrast at higher temperatures both $\mathrm{Cu}$ and $\mathrm{Ag}$ UPD result in ordered, smooth thin film structures.

Although the results for the different systems show the similar trend of enhanced surface ordering at higher temperatures, the underlying mechanism might differ from system to system. The $\mathrm{Cu}$ UPD shows a higher ordering at high temperatures due to the formation of a phase which is frustrated at lower temperature. X-ray voltammetry, 
however, shows that the two other systems presented in this paper, differ in the ordering kinetics at different temperatures which is not due to frustration. The underlying mechanism of this change in kinetics is not totally understood. A lowering of the free energy of formation of the structure due to the effect of the entropy term $T \Delta S$ as well as a temperature dependence of the Gibbs free energy of formation related to, e.g. temperature induced solvation changes of the ions involved, will have to be considered in more detail.

We believe that these results open up new opportunities to establish correlations between temperature, structure, and surface reactivity that will be important for the future development of efficient energy conversion systems. For example, the stability of the metal catalysts used in low temperature hydrogen fuel cells is crucial to the longevity of operation. Given that such devices operate at temperatures that are significantly higher than ambient, a knowledge of the surface atomic structure, for example in bimetallic systems, would be invaluable. Furthermore, it has been demonstrated that temperature changes can strongly effect the concentration and mobility of surface defects during metal deposition. This opens up new routes to control thin film morphology during electrodeposition without the use of additives or other adsorbates. This will make it possible to probe the link between structure and reactivity on an adsorbate-free electrode, for example, enabling tuning of the surface reactivity without involving reaction-blocking species, such as carbon monoxide or other organic molecules. Finally, we note that non-aqueous electrolytes, i.e. ionic liquids and organic electrolytes, are becoming of increasing importance in many electrochemical processes and these electrolytes present a significantly larger accessible temperature range compared to aqueous electrolytes. Thus the methodologies for studying temperature 
changes and their influence on structure and reactivity are likely to be of growing interest.

\section{Acknowledgements}

We would like to thank the XMaS beamline staff for their support of the XMaS beamline, Paul Steadman for his support during the experiments at beamline ID3 at the ESRF, Nadia Leyarovska and Sungsik Lee for their support of BM12-BESSRC at the APS and Sean Brennan for all of the assistance that he has given with the operation of beamline 7-2 at SSRL. Use of the Stanford Synchrotron Radiation Lightsource, SLAC National Accelerator Laboratory, is supported by the U.S. Department of Energy, Office of Science, Office of Basic Energy Sciences under Contract No. DE-AC02-76SF00515. The XMaS beamline is an EPSRC mid-range facility managed by the University of Liverpool and the University of Warwick. We would like to thank a number of $\mathrm{PhD}$ students at the University of Liverpool for their contribution to this work. In chronological order they are Stuart Medway, Mark Gallagher, Ben Fowler, Dave Mercer, Mick Cormack, Alex Brownrigg and Naomi Sisson. YG, PT and CAL acknowledge the financial support of the EPSRC (UK). NMM is supported by the U.S. Department of Energy, Basic Energy Sciences, Materials Sciences and Engineering Division. YG acknowledges the financial support of the Royal Society (UK) through a University Research Fellowship. 


\section{References}

[1] M.F. Toney, B.M. Ocko, Atomic structure at electrode interfaces, Synchroton Radiation News, 6 (1993) 28-33.

[2] O.M. Magnussen, Ordered anion adlayers on metal electrode surfaces, Chem.Rev., 102 (2002) 679-725.

[3] D.M. Kolb, An atomistic view of electrochemistry, Surface Science, 500 (2002) 722-740.

[4] C.A. Lucas, N.M. Markovic, in: R.C. Alkire, D.M. Kolb, J. Lipkowski, P.N. Ross (Eds.) Advances in Electrochemical Science and Engineering, 2006.

[5] Solid-Liquid Interfaces: macroscopic phenomena, microscopic understanding, Springer, New York, 2002.

[6] D.M. Kolb, Reconstruction phenomena at metal-electrolyte interfaces, Prog. Surf.Sci., 51 (1996) 109-173.

[7] M.F. Toney, J.N. Howard, J. Richter, G.L. Borges, J.G. Gordon, O.R. Melroy, D.G. Wiesler, D. Yee, L.B. Sorensen, Voltage-dependent ordering of water molecules at an electrolyte-electrolyte interface, Nature, 368 (1994) 444-446.

[8] S. Huemann, N.T.M. Hai, P. Broekmann, K. Wandelt, H. Zajonz, H. Dosch, X-ray diffraction and STM study of reactive surfaces under electrochemical control: $\mathrm{Cl}$ and I on $\mathrm{Cu}(100)$, J.Phys.Chem.B, 110 (2006) 24955-24963.

[9] Y. Gründer, D. Kaminski, F. Golks, K. Krug, J. Stettner, O.M. Magnussen, A. Franke, J. Stremme, E. Pehlke, Reversal of chloride-induced $\mathrm{Cu}(001)$ subsurface buckling in the electrochemical environment: An in situ surface x-ray diffraction and density functional theory study, Phys.Rev.B, 81 (2010).

[10] C.A. Lucas, N. Markovic, In-situ spectroscopic studies of adsorption at the Electrode and Electrocatalysis, in: S.G. Sun, P.A. Christensen, A. Wieckowski (Eds.), Elsevier, 2007.

[11] V.R. Stamenkovic, B. Fowler, B.S. Mun, G. Wang, P.N. Ross, C.A. Lucas, N.M. Markovic, Improved oxygen reduction activity on $\mathrm{Pt}_{3} \mathrm{Ni}(111)$ via increased surface site availability, Science, 315 (2007) 493-497.

[12] C.A. Lucas, P. Thompson, Y. Grunder, N.M. Markovic, The structure of the electrochemical double layer: $\mathrm{Ag}(111)$ in alkaline electrolyte, Electrochem. Comm., 13 (2011) 1205.

[13] C.A. Lucas, N.M. Markovic, Structure Relationships in Electrochemical Reactions, in: A.J. Bard, M. Stratmann, E.J. Calvo (Eds.) Encyclopedia of Electrochemistry, Interfacial Kinetics and Mass Transport, Wiley VCH, 2003.

[14] F. Zaera, Probing Liquid/Solid Interfaces at the Molecular Level, Chemical Review, 112 (2012) 2920-2986.

[15] C.A. Lucas, P. Thompson, M. Cormack, A. Brownrigg, B. Fowler, D. Strmcnik, V. Stamenkovic, J. Greeley, A. Menzel, H. You, N.M. Markovic, Temperature-Induced Ordering of Metal/Adsorbate Structures at Electrochemical Interfaces, Journal of the American Ceramic Society, 131 (2009) 7654-7661.

[16] P.A. Thiel, P.J. Estrup, in: A.T. Hubbard (Ed.) Handbook of Surface Imaging and Visualization, CRC Press: Boca Raton, 1995.

[17] K. Itaya, In situ scanning tunneling microscopy in electrolyte solutions, Prog.Surf.Sci., 58 (1998) 121-247.

[18] V.A. Marichev, Partial charge transfer during anion adsorption: Methodological aspects, Surface Science Reports, 56 (2005) 277-324. 
[19] P. Rodriguez, Y. Kwon, M.T.M. Koper, The promoting effect of adosrbed carbon monoxide on the oxidation of alcohols on a gold catalyst, Natue Chemistry, 4 (2012) 177-182.

[20] N. Markovic, H.A. Gasteiger, C.A. Lucas, I.M. Tidswell, P.N. Ross, The effect of chloride on the underpotential deposition of copper on Pt(111): AES, LEED, RRDE and x-ray scattering studies, Surf.Sci., 335 (1995) 91-100.

[21] S. Ferrer, F. Comin, Surface diffraction beamline at ESRF, Review of Scientific Instruments, 66 (1995) 1674-1676.

[22] S.D. Brown, L. Bouchenoire, D. Bowyer, J. Kervin, D. Laundy, M.J. Longfield, D. Mannix, D.F. Paul, A. Stunault, P. Thompson, M.J. Cooper, C.A. Lucas, W.G. Stirling, The XMaS beamline at ESRF: instrumental developments and high resolution diffraction studies, Journal of Synchrotron Radiation, 8 (2001) 1172-1181.

[23] D.F. Paul, M.J. Cooper, W.G. Stirling, Design of an X-ray beamline on a bending magnet at the ESRF for magnetic and high resolution diffraction, Review of Scientific Instruments, 66 (1995) 1741-1744.

[24] C.A. Lucas, N.M. Markovic, P.N. Ross, Surface Structure and Relaxation at the Pt(110)/Electrolyte Interface, Phys.Rev.Lett., 77 (1996) 4922-4925.

[25] X. Gao, A. Hamelin, M.J. Weaver, Atomic relaxation at ordered electrode surfaces probed by scanning tunneling microscopy: $\mathrm{Au}(111)$ in aqueous solution compared with ultrahigh-vacuum environments, The Journal of Chemical Physics, 95 (1991) 69936996.

[26] M.A. Schneeweiss, D.M. Kolb, D. Liu, D. Mandler, Anodic Oxidation of Au(111), Canadian Journal of Chemistry, 75 (1997) 1703-1709.

[27] J. Wang, B.M. Ocko, A.J. Davenport, H.S. Isaacs, In situ X-ray-diffraction and reflectivity studies of the $\mathrm{Au}(111) /$ electrolyte interface: Reconstruction and anion adsorption, Physical Review B, 46 (1992) 10321-10338.

[28] J. Perdereau, J.P. Biberian, G.E. Rhead, Adsorption and surface alloying of lead monolayers on (111) and (110) faces of gold, J. Phys, F 4 (1974) 1978.

[29] A.R. Sandy, S.G.J. Mochrie, D.M. Zehner, K.G. Huang, D. Gibbs, Structure and phases of the Au(111) surface: X-ray-scattering measurements, Physical Review B, 43 (1991) 4667.

[30] J.V. Barth, H. Brune, G. Ertl, R.J. Behm, Scanning tunneling microscopy observations on the reconstructed $\mathrm{Au}(111)$ surface: Atomic structure, long-range superstructure, rotational domains, and surface defects, Physical Review B, 42 (1990) 9307.

[31] Y. Wang, N.S. Hush, J.R. Reimers, Simulation of the Au(111)-(22 x sqrt(3)) surface reconstruction, Physical Review B (Condensed Matter and Materials Physics), 75 (2007) 233416.

[32] T.J. Schmidt, V. Stamenkovic, M. Arenz, N.M. Markovic, P.N. Ross, Electrooxidation of $\mathrm{H} 2, \mathrm{CO}$ and $\mathrm{H} 2 / \mathrm{CO}$ on well characterized $\mathrm{Au}$ (111)-Pd surface alloys, Electrochim. Acta, 47 (2002).

[33] I.M. Tidswell, N.M. Markovic, C.A. Lucas, P.N. Ross, In-situ X-ray diffraction study of $\mathrm{Au}(001)$ reconstruction in alkaline and acidic electrolytes, Physical Review B, 47 (1993) 16542-16553.

[34] B.B. Blizanac, C.A. Lucas, M.E. Gallagher, M. Arenz, P.N. Ross, N.M. Markovic, Anion adsorption, $\mathrm{CO}$ oxidation, and oxygen reduction on a $\mathrm{Au}(001)$ surface: The $\mathrm{pH}$ effect, Journal of Physical Chemistry B, 108 (2004) 625-634. 
[35] M.A. Pasquale, F.J.R. Nieto, A.J. Arvia, In-situ scanning tunneling microscopy topography changes of gold (111) in aqueous sulfuric acid produced by electrochemical surface oxidation and reduction and relaxation phenomena, Surface Review and Letters, 15 (2008) 847-865.

[36] N. Furuya, M. Shibata, Structural changes at various Pt single crystal surfaces with potential cycles in acidic and alkaline solutions, J.Electroanal.Chem., 467 (1999) 85-91.

[37] J. Honbo, S. Sugawara, K. Itaya, A detailed in situ scanning tunneling microscopy study of single crystal planes of $\mathrm{Au}(111)$ in aqueous solutions, Anal.Chem., 62 (1990) 2424-2429.

[38] M. Haruta, T. Kobayashi, H. Sano, M. Yamada, Novel catalysis of gold deposited on metal oxides, Chem. Lett, 16 (1987) 405-408.

[39] M. Haruta, Catalysis: Gold rush, Nature, 437 (2005) 1098.

[40] A.S.K. Hashmi, G.J. Hutchings, Gold Catalysis, Angewandte Chemie-International Edition, 45 (2006) 7896-7936.

[41] P. Rodriguez, J.M. Feliu, M.T.M. Koper, Unusual adsorption state of carbon monoxide on single-crystalline gold electrodes in alkaline media, Electrochem. Comm., 11 (2009) 1105-1108.

[42] P. Rodriguez, A.A. Koverga, M.T.M. Koper, Carbon Monoxide as a Promoter for its own Oxidation on a Gold Electrode, Angew.Chem., 49 (2010) 1241-1243.

[43] P. Rodriguez, N. Garcia-Araez, M.T.M. Koper, Self-promotion mechanism for CO electrooxidation on gold, Physical Chemistry Chemical Physics, 12 (2010) 9373-9380.

[44] G. Harlow, Y. Grunder, N. Sisson, P. Thompson, E. Cocklin, C.A. Lucas, in preparation

[45] N. Markovic, P.N. Ross, Effect of anions on the underpotential deposition of $\mathrm{Cu}$ on $\mathrm{Pt}(111)$ and $\mathrm{Pt}(100)$ surfaces, Langmuir, 9 (1993) 580-590.

[46] J. Gómez, H.S. Yee, G.M. Bommarito, J.M. Feliu, H.D. Abruña, Anion effects and the mechanism of Cu UPD on Pt(111): X-ray and electrochemical studies, Surf.Sci., 331 (1995) 101-109.

[47] E. Herrero, L.J. Buller, H.D. Abruna, Underpotential Deposition at Single Crystal Surfaces of Au, Pt, Ag and Other Materials, Chemical Review, 101 (2001) 1897-1930.

[48] E. Herrero, S. Glazier, H.D. Abruña, X-ray and electrochemical studies of Cu UPD on $\mathrm{Au}(111)$ single-crystal electrodes in the presence of bromide, J.Phys.Chem.B, 102 (1998) 9825-9833.

[49] C.A. Lucas, I.M. Tidswell, N.M. Markovic, P.N. Ross, In-situ X-ray Scattering Study of the Pt(111)-Solution Interface: ordered Anion Structures and their Influence on Copper Underpotential Deposition, Physica B, 221 (1996) 245-250.

[50] N.M. Markovic, C.A. Lucas, H. Gasteiger, P.N. Ross, The Structure of Adsorbed Bromine Concurrent with the Underpotential Deposition of $\mathrm{Cu}$ on $\mathrm{Pt}(111)$, Surface Science, 372 (1997) 239-254.

[51] C.A. Lucas, M. N.M., P.N. Ross, Underpotential Deposition of $\mathrm{Cu}$ on $\operatorname{Pt}(001)$ : Interface Structure and the Influence of Adsorbed Bromide, Physical Review B, 57 (1998) 13184-13191.

[52] C. Sánchez, E.P.M. Leiva, Cu underpotential deposition on $\mathrm{Au}(111)$ and $\mathrm{Au}(100)$. $\mathrm{Can}$ this be explained in terms of energetics of the $\mathrm{Cu} / \mathrm{Au}$ system?, Electrochim.Acta, 45 (1999) 691-697.

[53] C.G. Sanchez, E.P.M. Leiva, J. Kohanoff, Relevance of heterometallic binding energy for metal underpotential deposition, Langmuir, 17 (2001) 2218-2227. 
[54] M.C. Gimenez, A.J. Ramirez-Pastor, E.P.M. Leiva, A model for underpotential deposition in the presence of anions, J. Chem. Phys., 132 (2010).

[55] E. Herrero, S. Glazier, L.J. Buller, H.D. Abruña, X-ray and electrochemical studies of $\mathrm{Cu}$ UPD on single crystal electrodes in the presence of bromide: comparison between $\mathrm{Au}(111)$ and $\mathrm{Pt}(111)$ electrodes, J.Electroanal.Chem., 461 (1999) 121-130.

[56] I.M. Tidswell, C.A. Lucas, N. Markovic, P.N. Ross, Surface structure determination using anomalous $\mathrm{X}$-ray scattering: underpotential deposition of copper on Pt(111), Phys.Rev.B, 51 (1995) 10205-10208.

[57] F. Golks, J. Stettner, Y. Gründer, K. Krug, J. Zegenhagen, O.M. Magnussen, Anomalous potential dependence in homoepitaxial $\mathrm{Cu}(001)$ electrodeposition: an in situ surface x-ray diffraction study, Physical Review Letters, 108 (2011) 256101.

[58] G. Jerkiewicz, F. Perreault, Z. Radovic-Hrapovic, Effect of Temperature Variation on the Under-Potential Deposition of Copper on Pt(111) in Aqueous H2SO4, Journal of Physical Chemistry C, 113 (2009) 12309.

[59] T. Kondo, Y. Morita, M. Okamura, T. Saito, K. Uosaki, In situ structural study on underpotential deposition of $\mathrm{Ag}$ on $\mathrm{Au}(111)$ electrode using surface X-ray scattering technique Journal of Electroanalytical Chemistry, 532 (2002) 201-205.

[60] J.D. Snyder, J.D. Erlebacher, Electrochemical Measurement of the Surface Alloying Kinetics of Underpotentially Deposited Ag on Au (111), Langmuir, 25 (2009) 9596-9604.

[61] T. Kondo, S. Takakusagi, K. Uosaki, Stability of underpotentially deposited Ag layers on a $\mathrm{Au}(111)$ surface studied by surface X-ray scattering, Electrochem. Comm., 11 (2009) 804-807.

[62] M.F. Toney, J.N. Howard, J. Richer, G.L. Borges, J.G. Gordon, O.R. Melroy, Electrochemical deposition of copper on a gold electrode in sulfuric acid: resolution of the interfacial structure, Phys.Rev.Lett., 75 (1995) 4472-4475.

[63] N. Sisson, D. Hesp, E. Hynes, M. Darlington, P. Thompson, Y. Grunder, C.A. Lucas, The structure and stability of underpotentially deposited Ag on $\mathrm{Au}(111)$ in alkaline electrolyte, to be submitted, (2014). 


\section{Figure Captions}

Figure 1 A schematic diagram of the temperature-controlled x-ray electrochemical cell.

Figure 2 (a) In plane diffraction along the $\langle 1,1,0\rangle$ direction, measured at a potential of $-0.9 \mathrm{~V}$, through the $(0,1,0.52)$ position showing the peak due to the $(0,1, \mathrm{~L}) \mathrm{CTR}$ and the peak due to the Au surface reconstruction measured at low (blue) and high (red) temperature, $281 \mathrm{~K}$ and $313 \mathrm{~K}$ respectively in $0.1 \mathrm{M} \mathrm{KOH}$. The solid lines are fits of a double Lorentzian lineshape to the data (b) The x-ray voltammetry (XRV) measured at the $(0,1,0.52)$ CTR position at low (blue lines) and high (red lines) temperature. The arrows indicate the sweep direction and the scan rate was $2 \mathrm{mV} / \mathrm{sec}$.

Figure 3 (a) Schematic of the surface plane of $\mathrm{Au}(111)$ reciprocal space showing the position of the SXS peaks that arise due to the $\mathrm{Cu}-\mathrm{Br}$ adlayer. The arrow indicates the direction in which the peak moves. (b) Scans along the $\langle 1,1,0\rangle$ direction through the $\mathrm{Cu}-\mathrm{Br}$ adlayer peak at $(0.75,0.75,0.2)$ measured at $70 \mathrm{mV}$ (triangles) and $370 \mathrm{mV}$ (squares) at low (blue) and high (red) temperatures, $279 \mathrm{~K}$ and $311 \mathrm{~K}$ respectively. The solid lines are fits of a single Lorentzian lineshape to the data.

Figure 4 (a) XRVs measured at high (red) and low (blue) temperatures measured at the position $(0.745,0.745,0.2)$. The arrows indicate the sweep directions and the scan rate was $2 \mathrm{mV} / \mathrm{sec}$. Results from Lorentzian fits to scans such as that shown in Figure 3(b) as the potential was stepped negatively across the potential range indicated plotting (b) the peak position in $\mathrm{H}, \mathrm{K}$, (c) the domain size obtained from the FWHM of the peak and (d) the peak amplitude.

Figure 5 (a) XRV data for the $\mathrm{Au}(111)$ electrode measured at $(0,0,1.52)$, an anti-Bragg position on the specular CTR, during UPD of Ag monolayer in $0.05 \mathrm{M} \mathrm{H}_{2} \mathrm{SO}_{4}+1 \mathrm{mM}$ 
$\mathrm{Ag}_{2} \mathrm{SO}_{4}$ electrolyte at high (red) and low (blue) temperatures, $311 \mathrm{~K}$ and $279 \mathrm{~K}$ respectively. The sweep rate was $5 \mathrm{mV} / \mathrm{sec}$. (b) The specular CTR data, (0, 0, L), measured at 0.2 V after UPD at high (red) and low (blue) temperature. Solid lines are fits to the data according to the simple structural model described in the text. (c) The measured (data points) and calculated (solid line) ratio of the two CTR data sets shown in (b). 


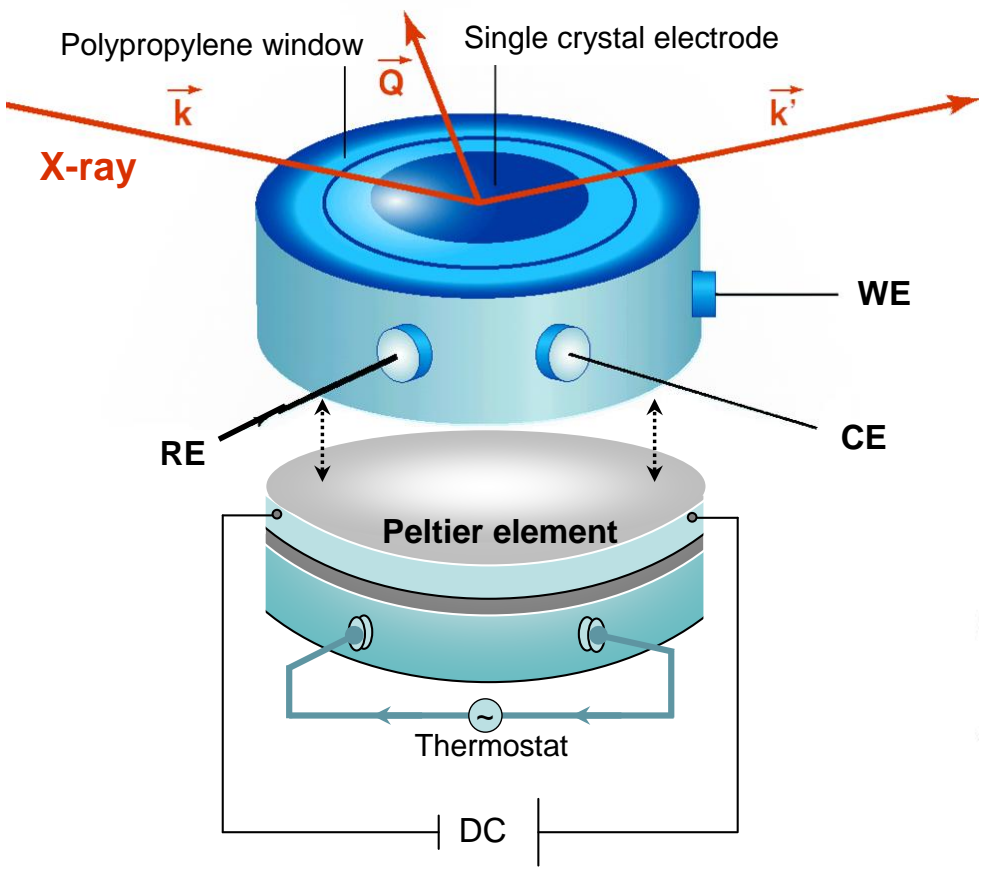

Figure 1 


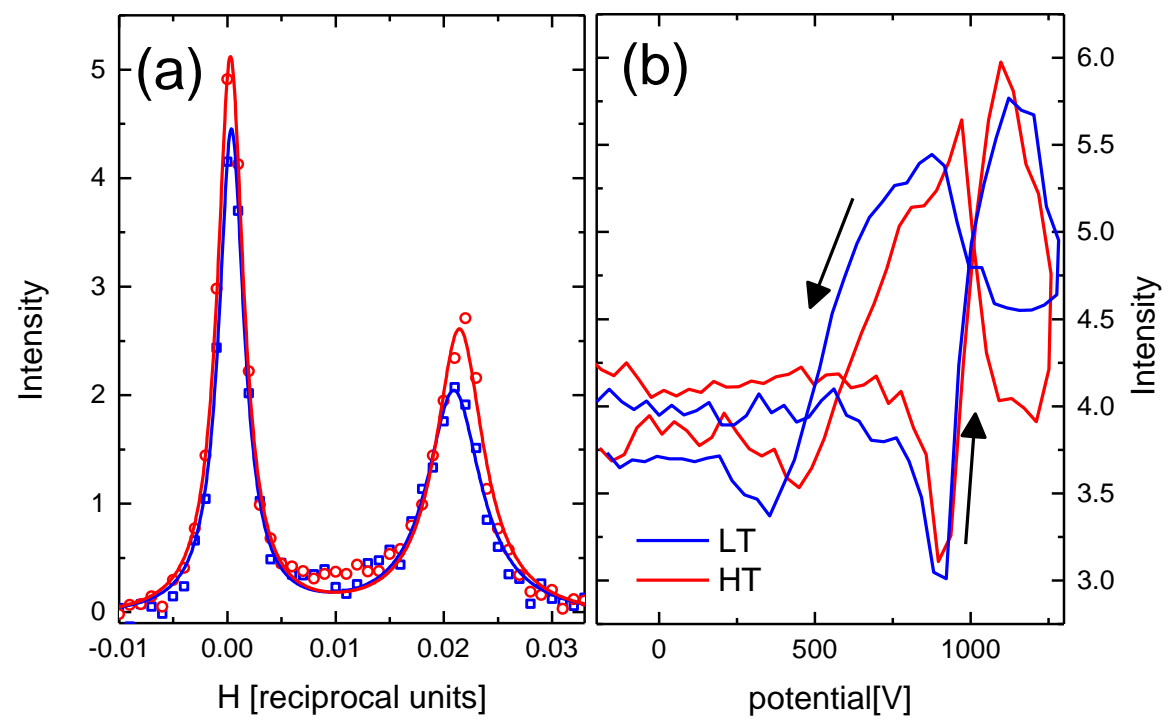

Figure 2 

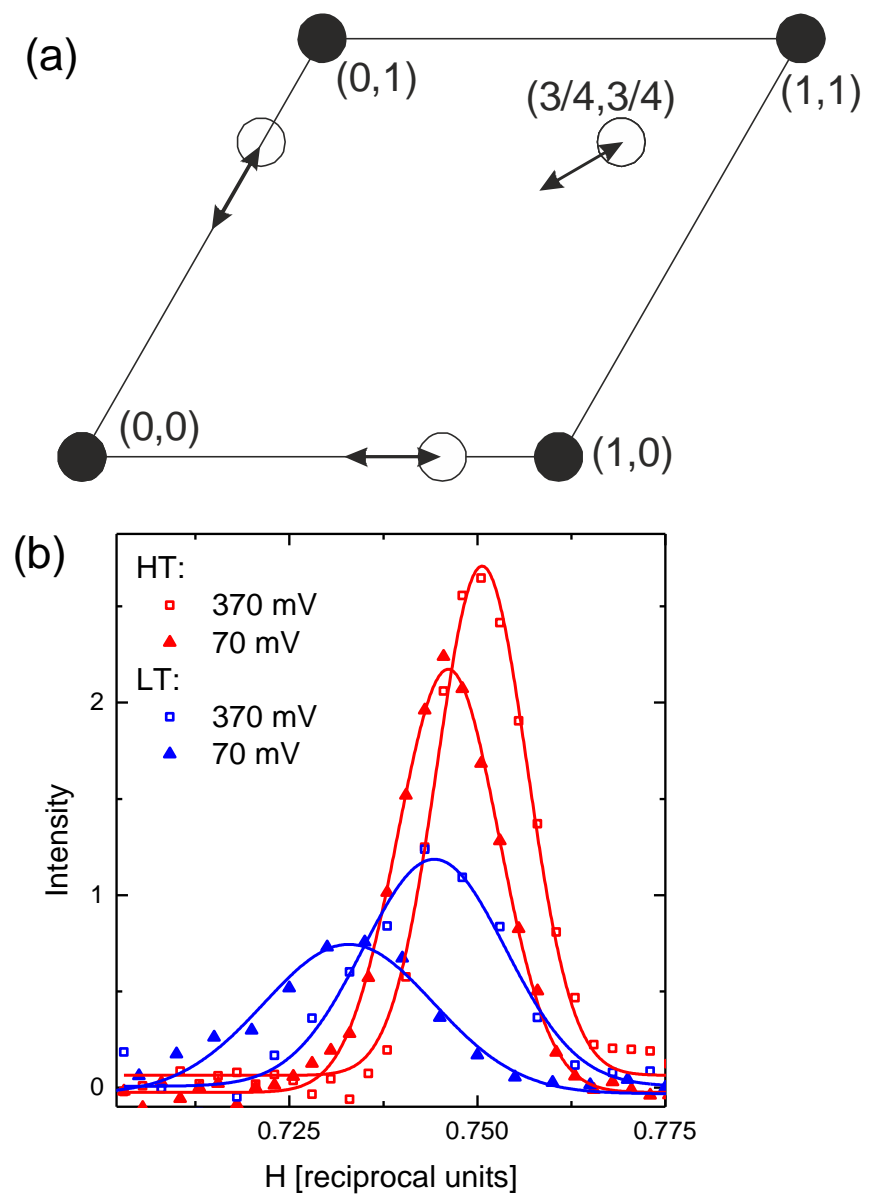

Figure 3 


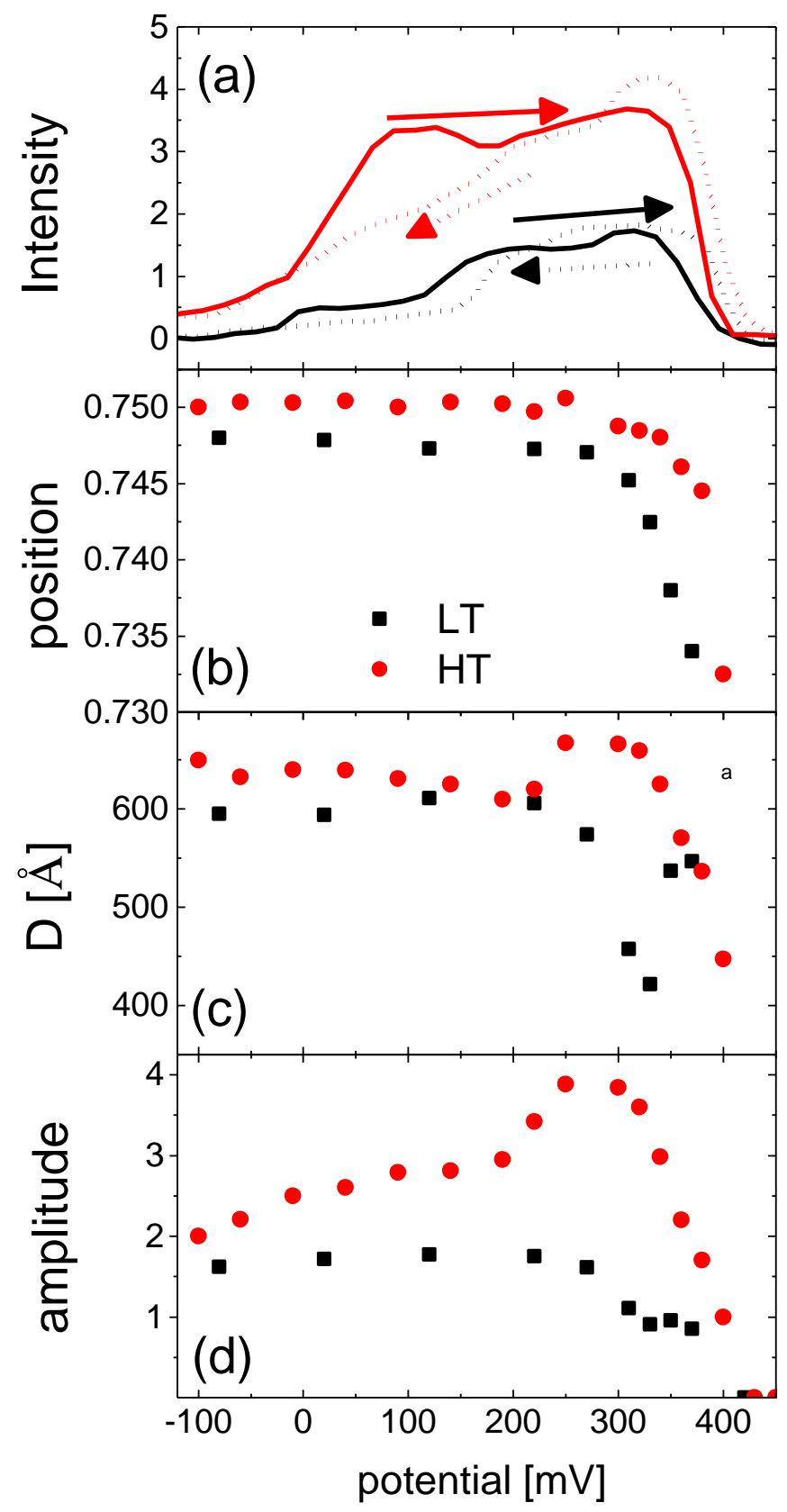

Figure 4 


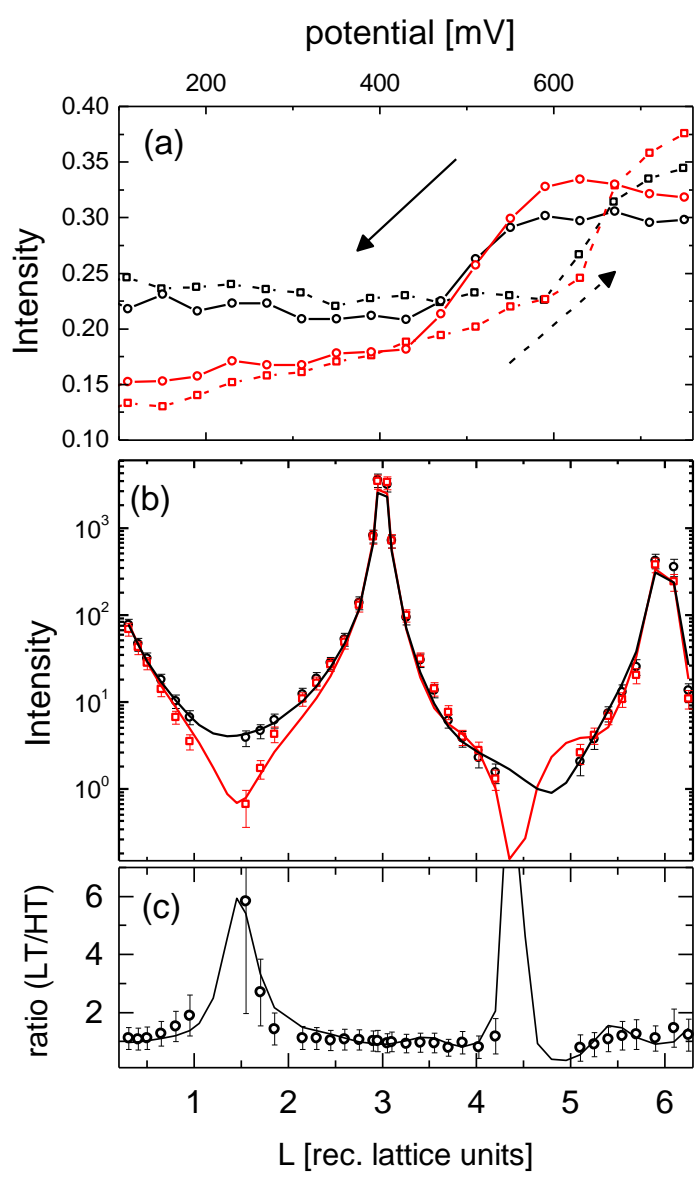

Figure 5 